\title{
Iterative Reweighted Least Squares Approach to Interference Alignment
}

\author{
Mohamed Rihan ${ }^{1}$, Maha Elsabrouty ${ }^{1}$, Said Elnouby ${ }^{2}$, Hossam Shalaby ${ }^{1,2}$, Osamu Muta ${ }^{3}$, and Hiroshi Furukawa ${ }^{4}$ \\ 1: Department of Electronics and Communications Engineering \\ Egypt-Japan University of Science and Technology(E-JUST), Alexandria, Egypt. \\ Email: mohamed.elmelegy@ejust.edu.eg, maha.elsabrouty@ejust.edu.eg, and hossam.shalaby@ejust.edu.eg \\ 2: Department of Electrical Engineering, University of Alexandria, Alexandria, Egypt. \{Email : saidelnoubi@yahoo.com\} \\ 3: Center for Japan-Egypt Cooperation in Science and Technology, Kyushu University, Japan. \\ 4: Graduate school of information Science and Electrical Engineering, Kyushu University, Japan
}

\begin{abstract}
This paper investigates the interference alignment (IA) solution for a $K$-user static flat-fading multiple input multiple output (MIMO) interference channel. Optimal users' precoders and postcoders are designed through a rank constraint rank minimization (RCRM) framework with IA conditions inserted within the constraints and the cost function of a complex matrix optimization problem. With RCRM formulation, the interference is forced to span the lowest dimensional subspace possible, under the condition that the useful signal subspaces span all available spatial dimensions. Using the recent advances in matrix completion theory and low rank matrix recovery theory, we propose an Iterative Reweighted Least Squares (IRLS) approach to IA. Through this approach, we provide an adequate relaxation for the rank function which in some cases attain the same results obtained using the standard nuclear norm with lower elapsed time per iteration and lower number of iterations and in some cases perform better than any of the previous approaches.
\end{abstract}

Keywords-Iterative Reweighted Least Square (IRLS), Degrees of freedom (DoF), Interference alignment, Interference channel, MIMO, Alternative minimization.

\section{INTRODUCTION}

Traditionally, interference is considered as a major limiting factor when designing any wireless communication system. Interference alignment was introduced as a solution to the interference problem by showing that with any $K$ users in the network, every user still have the ability to achieve half the capacity of the interference free case [1]. IA can be viewed as a cooperative interference management strategy through which the transmitters cooperatively design their transmitted signals in the multidimensional space such that the interference observed at the receivers occupies only a portion of the full signalling space [1][2]. As an efficient transmission technology, IA has a wide application in modern advanced wireless communication systems such as femto-cell networks, cognitive radio networks, and ad-hoc networks [4]-[8].

Communication in the presence of interference can be analysed using a model called the interference channel. This interference channel consists of multiple transmitters who wish to communicate with their respective receivers. Assuming the transmitters share the same time and frequency resources, each transmission creates interference at the unintended receivers [3]. IA can achieve sum capacity for the $K$-user interference channel that scales linearly and without bound with the number of users, in high signal-to-noise ratio(SNR) regime. Intuitively,
IA jointly optimizes precoding matrices for all transmitters, so that all the interference at one receiver fall into a reduced dimensional subspace. Then by multiplying postcoding matrix orthogonal to this subspace, the intended receiver can extract the desired signals without interference. In order to satisfy perfect IA, it is required for some conditions to be realized. These conditions include: all interference signals must be eliminated, all the signal subspaces must be full rank, and the precoders and postcoders must be orthogonal. However the achievement of such conditions is not a simple problem unless some special cases as explained in [1] and [9] where closedform solutions are introduced. As an alternative to closedform solutions, there are some iterative algorithms that was introduced in the literature, such as [10]-[13].

Recently, the authors in [14] has proposed a rank constraint rank minimization (RCRM) framework in order to obtain near optimal IA solutions and achieve the desirable Degree of freedom (DoF) per user. This framework poses full-rank constraints on the useful signal subspaces and minimize the rank of the interference subspaces. The full-rank constraints guarantee that useful signal subspaces span all available spatial dimensions. The rank minimization forces the interference subspaces to squeeze to the minimum dimensions possible. In [14], the authors introduce a convex relaxation of the RCRM problem inspired by recent results in low-rank matrix completion theory. These relaxations rely on approximating rank with the standard nuclear norm. Depending on these relaxations, they show that the convex envelope of the sum of the ranks of the interference matrices is the normalized sum of their corresponding nuclear norms and replace the rank constraints with asymptotically equivalent and tractable ones. By careful inspection of this approximation, it was shown that nuclear norm approximation is not tight and accurate enough for approximating the rank function, because it is sensitive to the influence of its magnitude. In [15], the authors proposed a family of Iterative Reweighed Least Squares (IRLS-q) (with $0 \leq q \leq 1$ ), as a computationally efficient way to improve over the performance of nuclear norm minimization.

In this paper, we propose applying IRLS approach to IA for static flat-fading K-user MIMO interference channels. Motivated by maximizing the average DoF per user, we use the RCRM problem developed in [14]. Unlike [14], an alternative solution to the non-convexity of the RCRM problem, namely IRLS-q based approach, is developed to approximate the cost 
function as the weighted Frobenius norm of the interference matrix. The proposed IA algorithm exhibits fast convergence (low number of iterations) and low complexity where the iterations are based on weighted Frobenius norm instead of nuclear norm that requires singular value decomposition (SVD) to calculate. Then, the optimum precoding and postcoding matrices are obtained using alternative minimization. The efficiency of the proposed algorithm is evaluated in terms of both the average sum rate and the average DoF per user which is useful when targeting higher DoF per user.

The remainder of the paper is organized as follow. In section II, description of the system model and its mathematical manipulation is presented. Section III explains the RCRM framework for interference alignment. The Iterative Reweighted Least Squares algorithm relaxation is explained in Section IV . The simulation results are presented and discussed in Section V. Finally, the paper is concluded in Section VI.

Notations: matrices and vectors are denoted by boldface upper case symbols and boldface lower case symbols respectively. An identity matrix of size $N \times N$ is simply denoted by $\mathbf{I}_{N}$. Positive semi-definiteness of the matrix $\mathbf{A}$ is depicted using $\mathbf{A} \geq 0 . \mathbf{A}^{T}$ and $\mathbf{A}^{H}$ refer to the transpose and the hermitian of matrix A respectively. $\mathbb{C}^{m \times n}$ is used to describe the complex space of $m \times n$ matrices. $\|\mathbf{A}\|_{F}$ and $\operatorname{tr}\{\mathbf{A}\}$ are used to refer to the Frobenius norm and the trace of the matrix A. $\|\mathbf{A}\|_{*}$ refers to the nuclear norm of the matrix $\mathbf{A}$ which is equivalent to the sum of its corresponding singular values. $\sigma_{i}(\mathbf{A})$ and $\sigma_{\min }(\mathbf{A})$ represents the $i_{t h}$ largest singular value and the minimum singular value of $\mathbf{A}$ respectively. The operation $[a\rceil^{+}$denotes maximum(a,0). Finally, $S V D(\mathbf{A})$ and $Q R(\mathbf{A})$ denotes the singular value decomposition and the $Q R$ factorization of matrix $\mathbf{A}$ respectively.

\section{System Model And Perfect Interference Alignment Conditions}

\section{A. System Model}

Consider a static flat-fading $K$-user MIMO interference channel $\left(M_{t} \times M_{r}, d\right)^{K}$ as shown in Fig.1, where each transmitter equipped with $M_{t}$ antennas, and each receiver equipped with $M_{r}$ antennas with each user pair wishes to achieve $d$ DoF. Suppose that $x_{k} \in \mathbb{C}^{d \times 1}$ denotes the transmit signal vector of the $k^{t h}$ user, $\forall k \in K$, with power covariance as $E\left(\mathbf{x}_{k} \mathbf{x}_{k}^{T}\right)=(P / d) \mathbf{I}_{d}$, where $P$ is the total transmitted power of each user. After receiving the signal $\mathbf{y}_{k}$, the $k^{t h}$ receiver multiplies the corresponding postcoding matrix $\mathbf{W}_{k}^{H}$ to it on the left, which is expressed as in equation (1):

$$
\mathbf{W}_{k}^{H} \mathbf{y}_{k}=\underbrace{\mathbf{W}_{k}^{H} \mathbf{H}_{k, k} \mathbf{F}_{k} \mathbf{x}_{k}}_{\text {useful signal }}+\underbrace{\mathbf{W}_{k}^{H} \sum_{l=1, l \neq k}^{K} \mathbf{H}_{k, l} \mathbf{F}_{l} \mathbf{x}_{l}}_{\text {Interference }}+\underbrace{\mathbf{W}_{k}^{H} \mathbf{v}_{k}}_{\text {Noise }}
$$

where $\mathbf{H}_{i, j} \in \mathbb{C}^{M_{r} \times M_{t}}$ denotes the channel matrix between the $j^{t h}$ transmitter and the $i^{t h}$ receiver. $\mathbf{F}_{k} \in \mathbb{C}^{M_{t} \times d}$ and $\mathbf{W}_{k} \in \mathbb{C}^{M_{r} \times d}$ represent the precoder and the postcoder of the $k^{t h}$ user, respectively. $\mathbf{v}_{k} \in \mathbb{C}^{M_{r} \times 1}$ denotes the zeromean complex additive white Gaussian noise vector with covariance matrix $\sigma_{k}^{2} \mathbf{I}_{M_{r}}$. In this paper, we assume without loss of generality that all the noises have the same covariance, that is $\sigma_{k}^{2}=\sigma^{2}, \forall k \in K$. If we assume that all signal subspaces span all available dimensions, we can say that $\operatorname{span}\left(\mathbf{W}_{k}^{H} \mathbf{H}_{k, k} \mathbf{F}_{k}\right)$ is the subspace where receiver $k$, $k \in K$, can search for the symbols transmitted by transmitter $k$. Additionally, $\operatorname{span}\left(\left\{\mathbf{W}_{k}^{H} \mathbf{H}_{k, l} \mathbf{F}_{l}\right\}_{l=1, l \neq k}^{K}\right)$ refers to the subspace where all interference signals are observed, where $\left\{\mathbf{S}_{l}\right\}_{l=1, l \neq k}^{K}$ denotes the horizontal concatenation of matrices $\mathbf{x}_{1}, \ldots, \mathbf{x}_{k-1}, \mathbf{x}_{k+1}, \ldots, \mathbf{x}_{K}$.

\section{B. Perfect Interference Alignment Conditions}

According to [1] and [14], the aim of IA is to design precoder matrices $\mathbf{F}_{k}$ and postcoder matrices $\mathbf{W}_{k}$, where $k \in K$, such that each receiver can decode its own signal by forcing interfering users to share a reduced-dimensional subspace. This can be translated to the conditions for perfect IA as in equations (2) and (3):

$$
\begin{aligned}
\mathbf{W}_{k}^{H} \mathbf{H}_{k, l} \mathbf{F}_{l} & =\mathbf{0}_{d \times d}, \quad \forall l \in K \backslash k \\
\operatorname{rank}\left(\mathbf{W}_{k}^{H} \mathbf{H}_{k, k} \mathbf{F}_{k}\right) & =d,
\end{aligned}
$$

Equation (2) can be rewritten as:

$$
\begin{aligned}
\mathbf{W}_{k}^{H} \mathbf{H}_{k, l} \mathbf{F}_{l} & =\mathbf{0}_{d \times d}, \quad \forall l \in K \backslash k \\
\Leftrightarrow\left[\left\{\mathbf{W}_{k}^{H} \mathbf{H}_{k, l} \mathbf{F}_{l}\right\}_{l=1, l \neq k}^{K}\right] & =\left[\mathbf{0}_{d \times d} \ldots . . \mathbf{0}_{d \times d}\right] \\
\Leftrightarrow \mathbf{W}_{k}^{H}\left[\left\{\mathbf{H}_{k, l} \mathbf{F}_{l}\right\}_{l=1, l \neq k}^{K}\right] & =\mathbf{0}_{d \times(K-1) d}
\end{aligned}
$$

According to equation (2), all interference subspaces enforced to have zero dimensions. Equation (3) enforces the useful signal to span all $d$ dimensions.

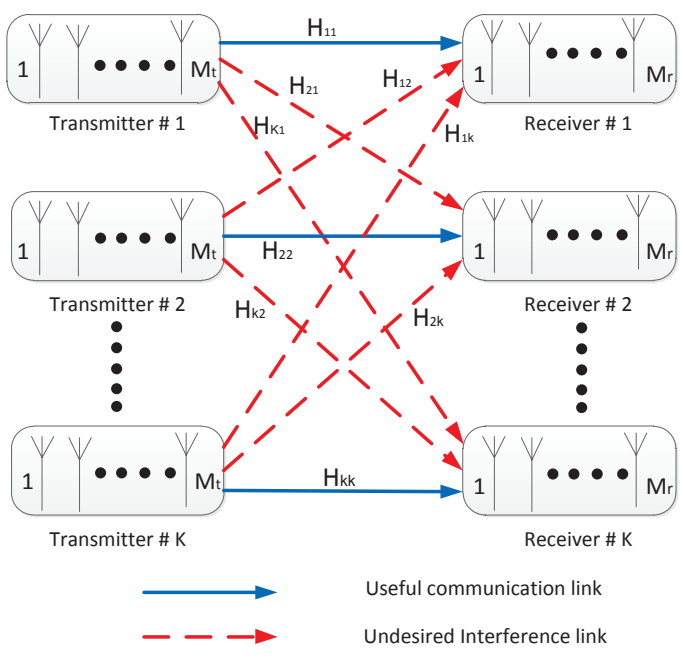

Fig. 1. K-user MIMO interference channel. 


\section{INTERFERENCE ALIGNMENT AS A RANK Constraint RANK Minimization}

The problem of finding precoding and postcoding matrices satisfying the IA conditions under arbitrary channel matrices is not an easy task. According to [16], the problem of checking the achievability of a certain DoF tuple $\left\{d_{1}, \ldots \ldots \ldots, d_{K}\right\}$, for $K$-user MIMO interference channel, is NP-hard. There are many approaches for achieving IA, even when perfect IA conditions are not feasible. One of these approaches is the leakage minimization approach, which aims to minimize the total interference leakage at each receiver [10]. However, minimizing the subspace spanned by the interference is related to minimizing the rank of the interference matrix rather than minimizing the energy of the interference signal. Another approach presented in [10], is the maximization of the signal to interference and noise ratio (SINR) at each receiver. However, it was shown in [14] that this approach gives lower performance than the leakage minimization in terms of both sum rate and DoF per user. Recently, in [14], the author established that the minimization of the sum of the interference dimensions under full-rank signal space constraints is equivalent to maximizing the sum multiplexing gain of a static flat-fading MIMO interference channel. This is referred to as RCRM problem.

Instead of minimizing the power of interference signal, the RCRM problem focuses on keeping the interference signals within a low dimensional subspace where the desired signal subspaces span all available spatial dimensions. RCRM is in general, an NP-hard problem and the rank as a cost function is a non-convex function. However, through convex relaxations it can be transformed to a problem that it is easy to solve. For example, [14] proposed to approximate the NP-hard rank function by using the standard nuclear norm. However, the recent result in minimum rank matrix recovery and sparse representation proposes a more efficient tractable relaxations to improve over the performance of nuclear norm minimization [15].

Let's defining the signal $\left(\mathbf{S}_{k}\right)$ and interference matrices $\left(\mathbf{J}_{k}\right)$ for all $k \in K$. They can be expressed as:

$$
\begin{aligned}
& \mathbf{S}_{k} \triangleq \mathbf{W}_{k}^{H} \mathbf{H}_{k, k} \mathbf{F}_{k} \in \mathbb{C}^{d \times d} \\
& \mathbf{J}_{k} \triangleq \mathbf{W}_{k}^{H}\left[\left\{\mathbf{H}_{k, l} \mathbf{F}_{l}\right\}_{l=1, l \neq k}^{K}\right] \in \mathbb{C}^{d \times(K-1) d}
\end{aligned}
$$

Equations (2) and (3) can be reformulated using rank functions as in equations (6) and (7) for all $k \in K$.

$$
\begin{aligned}
& \operatorname{rank}\left(\mathbf{S}_{k}\right)=d \\
& \operatorname{rank}\left(\mathbf{J}_{k}\right)=0
\end{aligned}
$$

Accordingly, the spatial DoF for any user $k$ can also be reformulated in terms of rank functions as in equation (8).

$$
d_{k} \triangleq\left\lceil\operatorname{rank}\left(\mathbf{S}_{k}\right)-\operatorname{rank}\left(\mathbf{J}_{k}\right)\right\rceil^{+}
$$

This result is possible, as the eigenvalues of the $\mathbf{S}_{k} \mathbf{S}_{k}^{H}$ and $\mathbf{J}_{k} \mathbf{J}_{k}^{H}$ scale linearly with $P$ for given precoding, postcoding, and channel matrices. The group of precoders and postcoders that will satisfy the IA conditions for perfect and imperfect cases will be obtained through solving the optimization problem in equation (9).

$$
\begin{aligned}
& \underset{\mathbf{F}_{k}, \mathbf{W}_{k}}{\operatorname{minimize}} \sum_{\substack{k=1 \\
\text { s.t. }}}^{K} \operatorname{rank}\left(\mathbf{W}_{k}^{H} \mathbf{H}_{k, k} \mathbf{F}_{k}\right)=d_{k}
\end{aligned}
$$

\section{ITERATIVE REWEIGHTED LEAST SQUARES INTERFERENCE ALIGNMENT APPROACH}

The problem of finding the minimum rank matrix arises in many engineering applications such as sparse recovery, compressive sensing, collaborative filtering, and matrix completion theory [15][17]. One of the tractable relaxations proposed for rank minimization problem is the nuclear norm heuristic, which is guaranteed to find the minimum rank matrix under suitable assumptions. In this paper, we propose using a more computationally efficient alternative in the RCRM framework than the standard nuclear norm heuristic. This alternative is the Iterative Reweighted Least squares (IRLS) algorithm which is used as a relaxation of the non-convex rank cost function in RCRM framework of IA. The IRLS-q algorithm can be viewed as the local minimization of certain smooth approximations to the rank function. When $q=1$, the IRLS$\mathrm{q}$ algorithm gives theoretical guarantees similar to those for the nuclear norm minimization. However, for $q<1$, IRLSq shows better empirical performance in terms of recovering low-rank matrices than the nuclear norm heuristic.

Our approach is divided into two parts. These two parts exploit the use of two different relaxations for the rank constraints and the rank of the cost function. The rank constraints as well as the cost function are non-convex sets and a relaxation to convex one is required. Two different relaxations are employed. As illustrated in [14], the rank constraints are relaxed by forcing the signal space matrices to be positive definite accompanied by putting a specific limiting factor on the minimum eigenvalue of each signal subspace $\mathbf{S}_{k}$ as shown in equation (10).

$$
\begin{aligned}
\operatorname{rank}\left(\mathbf{S}_{k}\right)=d_{k} \Longleftrightarrow & \mathbf{S}_{k}>\mathbf{0} \text { and } \lambda_{\min }\left(\mathbf{S}_{k}\right)>0 \\
\Longleftrightarrow & \lambda_{\min }\left(\mathbf{W}_{k}^{H} \mathbf{H}_{k, k} \mathbf{F}_{k}\right)>0 \\
& \quad \text { and } \quad \mathbf{W}_{k}^{H} \mathbf{H}_{k, k} \mathbf{F}_{k}>\mathbf{0}_{d \times d}
\end{aligned}
$$

for all $k \in K$, equation (10) can also be approximated to the closed sets in equation (11).

$$
\begin{aligned}
\operatorname{rank}\left(\mathbf{S}_{k}\right)=d_{k} \Longleftrightarrow & \lambda_{\min }\left(\mathbf{W}_{k}^{H} \mathbf{H}_{k, k} \mathbf{F}_{k}\right) \geq \epsilon \\
& \text { and } \mathbf{W}_{k}^{H} \mathbf{H}_{k, k} \mathbf{F}_{k} \geq \mathbf{0}_{d \times d}:
\end{aligned}
$$

where $\epsilon>0$.The rank cost function defined by equation (7) will be relaxed through the IRLS. Define the smooth Schatten$\mathrm{p}$ function as in equation(12). 


$$
\begin{aligned}
f_{q}\left(\mathbf{J}_{k}\right) & =\operatorname{Tr}\left(\mathbf{J}_{k}^{T} \mathbf{J}_{k}+\gamma \mathbf{I}\right)^{\frac{q}{2}} \\
= & \sum_{i=1}^{n}\left(\sigma_{i}^{2}\left(\mathbf{J}_{k}\right)+\gamma\right)^{\frac{q}{2}}
\end{aligned}
$$

This function is differentiable for $q>0$ and convex for $q \geqslant 1$. With $\gamma=0, f_{1}\left(\mathbf{J}_{k}\right)=\left\|\mathbf{J}_{k}\right\|_{*}$ with $\gamma=0$ and $q \rightarrow 0$ the function $f_{q}\left(\mathbf{J}_{k}\right) \rightarrow \operatorname{rank}\left(\mathbf{J}_{k}\right)$. So, it is of interest to consider the optimization problem in equation (13).

$$
\begin{array}{ll}
\underset{\mathbf{F}_{k}, \mathbf{W}_{k}}{\operatorname{minimize}} & \sum_{k=1}^{K} \operatorname{Tr}\left(\mathbf{M}_{q}^{k} \mathbf{J}_{k}^{T} \mathbf{J}_{k}\right) \\
\text { s.t. } & \mathbf{W}_{k}^{H} \mathbf{H}_{k, k} \mathbf{F}_{k} \geq \mathbf{0}_{d \times d}, \\
& \lambda_{\min }\left(\mathbf{W}_{k}^{H} \mathbf{H}_{k, k} \mathbf{F}_{k}\right) \geq \epsilon ; \quad \forall k \in K
\end{array}
$$

Where $\mathbf{M}_{q}^{k}$ is the weighting matrix at iteration $k$ with $I R L S$ parameter $q$, which is equivalent to $\left(\mathbf{J}_{k}^{T} \mathbf{J}_{k}+\gamma^{k} \mathbf{I}\right)^{1-\frac{q}{2}}$, with choosing $0<\gamma^{k+1} \leq \gamma^{k}, \gamma$ is called the regularization factor. We note that IRLS algorithm converges faster when the regularization parameter in the weighting matrix, $\gamma^{k}$, is chosen appropriately. Given $\mathbf{M}_{q}^{k}$, the optimization problem (13) can be solved using alternative minimization [11], alternating between which variables are held fixed and which are optimized. Listed below the IRLS which alternatively used to obtain the precoder and postcoder matrices.

$\overline{\text { Algorithm } 1\left\{\mathbf{F}_{l}\right\}_{l=1}^{K} \leftarrow \operatorname{IRLS}-q\left(\left\{\mathbf{W}_{l}\right\}_{l=1}^{K}\right)}$

Set $k=1$. Initialize $\mathbf{M}_{0}^{q}=\mathbf{I}, \gamma^{1}>0$

While not converged, do

$$
\begin{aligned}
& F_{k}=\operatorname{minimize}\left\{\operatorname{Tr}\left(\mathbf{M}_{k-1}^{q} \mathbf{J}^{T} \mathbf{J}\right)\right\} \\
& \text { s.t } \sigma_{\min }\left(\mathbf{W}_{k}^{H} \mathbf{H}_{k, k} \mathbf{F}_{k}\right) \leq \epsilon \\
& \mathbf{W}_{k}^{H} \mathbf{H}_{k, k} \mathbf{F}_{k} \leq 0 \\
& \mathbf{M}_{k+1}^{q}=\left(\mathbf{J}_{k}^{T} \mathbf{J}_{k}+\gamma^{k} \mathbf{I}\right)^{1-\frac{P}{2}} \\
& \text { Choose } 0<\gamma^{k} \leq \gamma^{k+1} \\
& \mathrm{k}=\mathrm{k}+1 ;
\end{aligned}
$$

End

By exchanging $\mathbf{F}_{k}$ and $\mathbf{W}_{k}$ in the IRLS-q algorithm we can obtain the optimization precoder and postcoding matrices. Each iteration of Algorithm 1 minimizes a weighted Frobenius norm of the matrix $\mathbf{J}$, since $\operatorname{Tr}\left(\mathbf{M}_{k-1}^{P} \mathbf{J}^{T} \mathbf{J}\right)=$ $\left\|\left(\mathbf{M}_{k-1}^{P}\right)^{1 / 2} \mathbf{J}\right\|_{F}^{2}$. While minimizing the Frobenius norm subject to some constraints doesn't lead to low-rank solutions in general, through a careful reweighting of this norm we show that Algorithm 1, indeed, produces low-rank solutions under suitable assumptions. Finally, the step-by-step IRLS based IA for the $k^{t h}$ user can be presented as follow:

1) Initialize $\left\{\mathbf{W}_{k=1}^{K}\right\}$, and $\mathbf{M}_{0}^{q} \quad \forall k=1: K$;

2) Solve the Iterative Reweighted Least Squares minimization problem in equation (13), for the $n^{\text {th }}$ iteration:

$$
\begin{array}{ll}
\text { a) } & \left\{\mathbf{F}_{l}\right\}_{l=1}^{K} \leftarrow \operatorname{IRLS}-q\left(\left\{\mathbf{W}_{l}\right\}_{l=1}^{K}\right) ; \\
\text { b) } \quad & \left\{\mathbf{W}_{l}\right\}_{l=1}^{K} \leftarrow \operatorname{IRLS}-q\left(\left\{\mathbf{F}_{l}\right\}_{l=1}^{K}\right) ;
\end{array}
$$

3) Terminate when $n$ attains a specified maximum number of iterations $N$.

Convergence in the proposed IRLS algorithm can be simply recognized by first expressing the iterations of IRLS as in equations $(14,15)$.

$$
\begin{aligned}
& \mathbf{J}^{k+1}=\operatorname{argmin} f^{q}\left(\mathbf{J}, \mathbf{M}^{k}, \gamma^{k}\right) \\
& \mathbf{M}^{k+1}=\operatorname{argmin} f^{q}\left(\mathbf{J}^{k+1}, \mathbf{M}, \gamma^{k}\right)
\end{aligned}
$$

Where $\operatorname{argmin}($.$) yields the new J^{k+1}$ using the computed values of $M^{k}$ and $\gamma^{k}$. Accordingly:

$$
\begin{aligned}
f^{q}\left(\mathbf{J}^{k+1}, \mathbf{M}^{k+1}, \gamma^{k+1}\right) \leq & f^{q}\left(\mathbf{J}^{k+1}, \mathbf{M}^{k}, \gamma^{k+1}\right) \\
\leq & f^{q}\left(\mathbf{J}^{k+1}, \mathbf{M}^{k}, \gamma^{k}\right) \\
& \leq f^{q}\left(\mathbf{J}^{k}, \mathbf{M}^{k}, \gamma^{k}\right)
\end{aligned}
$$

Equation (16) guarantees the convergence of the IRLS algorithm.

\section{Simulation Results}

In this section, we analyze the proposed IRLS-q algorithm using MATLAB simulations. We compare the proposed algorithm with its preceding algorithms, namely, nuclear norm heuristic [14], interference leakage minimization approach [10], and the SINR maximization (with and without QR decomposition) [18]. The notation $\left(M_{t} \times M_{r}, d\right)^{K}$ specifies a K-user interference channel, with each user equipped with $M_{t}$ transmitting antennas and $M_{r}$ receiving antennas with each user aiming to $d$ DoF. In this paper, we consider the $(8 \times 4, d=1,3)^{3}$ MIMO interference channel, for which the number of users is 3 users each equipped with 8 transmit antennas and 4 receive antennas. This MIMO interference channel will be tested under two values of the issued DoF per user $(1,3)$. The $(8 \times 4, d=1,3)^{3}$ system is a proper system, where it verify the rule $d \leq \frac{M_{t}+M_{r}}{K+1}$. In spite of being proper, this system is not guaranteed to achieve perfect IA. We assume that the transmit power is equally distributed between the columns of the precoding matrices, with each column get $\frac{10 \frac{P}{10}}{d_{k}}$. We choose the same range of $P$ as in [14], from $0 \mathrm{dBm}$ to $80 \mathrm{dBm}$. Additionally, we set the variance of the white Gaussian noise $\sigma_{k}^{2}=1 \mathrm{dBm}$. The channel is drawn from i.i.d real Gaussian distribution with zero mean and unit variance. The presented result is the average over 100 runs. The performance of different IA approaches is compared using two parameters. The first one is the average sum rate in bits $/ \mathrm{sec} / \mathrm{Hz}$ which is computed using equation (17).

$$
\begin{aligned}
\mathbf{R} & =\sum_{i=1}^{K} \frac{1}{2} \log \left(1+\operatorname{SIN} R_{k}\right) \\
& =\sum_{i=1}^{K} \frac{1}{2} \log \operatorname{det}\left(\mathbf{I}_{d}+\left(\mathbf{I}_{d}+\mathbf{J}_{k} \mathbf{J}_{k}^{H}\right)^{-1} \mathbf{S}_{k} \mathbf{S}_{k}^{H}\right)
\end{aligned}
$$


In equation (17), the second term inside the logdet function is the SINR for $k^{t h}$ user, where $\mathbf{S}_{k} \mathbf{S}_{k}^{H}$ is the signal power at transmitter $k, \mathbf{J}_{k} \mathbf{J}_{k}^{H}$ is the interference power at receiver $k$, and the noise power $\sigma_{k}^{2}$ for each user is assumed to be 1 , so the noise matrix is assumed to be identity matrix $\mathbf{I}_{d}$. The second parameter we used to judge the performance is the average DoF per user which is defined as the average number of interference free dimensions per user or the average multiplexing gain per user. The average DoF per user is calculated in simulation as the number of singular values of $S_{k}$ with value greater than $10^{-6}$, minus the number of singular values of $J_{k}$ that are greater than $10^{-6}$. The optimization problems in this paper are all solved using the CVX toolbox [19].

In our simulations, we select a specific number of iterations for each approach according to its convergence rate. In Fig.2, the sum rate versus $P$ for the $(8 \times 4, d=1)^{3}$ system is displayed. The leakage minimization and the SINR maximization approaches requires $10^{4}$ in order converge. However, the nuclear norm and our IRLS approaches requires much less number of iterations which are 5 and 3 iterations respectively. It is apparent that the proposed algorithm converges faster than any of the preceding approaches in terms of the number of iterations and the execution time for these iterations. According to Fig. 2 where $d=1$, our algorithm matches the one achieved by the nuclear norm approach at any $P$. Moreover, the SINR maximization approach (with and without $Q R$ ) slightly outperforms both the nuclear norm approach and our IRLS proposed approach at $P \leq 40 \mathrm{dBm}$ and matches them for $P \geq 40 \mathrm{dBm}$. In general, leakage minimization approach performance is the worst at all $P$.

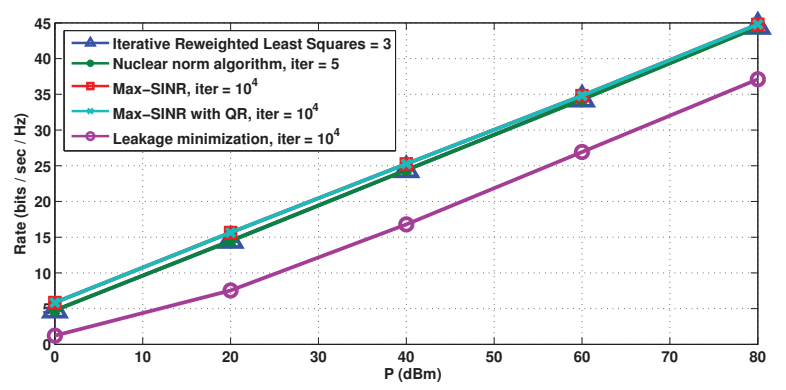

Fig. 2. Sum-rate versus $P$, for a 3-user MIMO interference channel, $M_{t}=$ $8, M_{r}=4, d=1$

As in Fig.3, the average DoF per user versus $P$ is plotted. It is apparent that the proposed IRLS algorithm, the nuclear norm and the leakage minimization approaches achieves in average one interference free dimension for each user at any $P$. While the number of interference free dimensions achieved by the maximum SINR approach varies according to the $P$ as shown in Fig. 3 where it gradually increases after $P=40 \mathrm{dBm}$. So, in order to obtain an improved performance in terms of both average DoF per user and the average sum rate for the $(8 \times 4, d=1)^{3}$ system, both the proposed IRLS algorithm and the nuclear norm algorithm can be used.

Fig. 4 and Fig. 5 are the average sum rate and average DoF per user for the $(8 \times 4, d=3)^{3}$ system respectively. As in Fig.4 with $P \leq 40 \mathrm{dBm}$, both the maximum SINR approach and the leakage minimization approach slightly outperform both

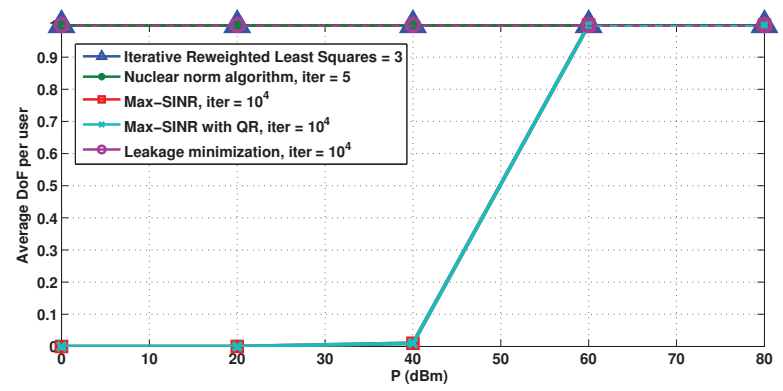

Fig. 3. Average DoF per user versus $P$, for a 3-user MIMO interference channel, $M_{t}=8, M_{r}=4, d=1$

our IRLS proposed algorithm and the nuclear norm approach. Beyond $P=40 \mathrm{dBm}$, the proposed IRLS algorithm and the nuclear norm approach have a matched performance which outperforms all other algorithms.

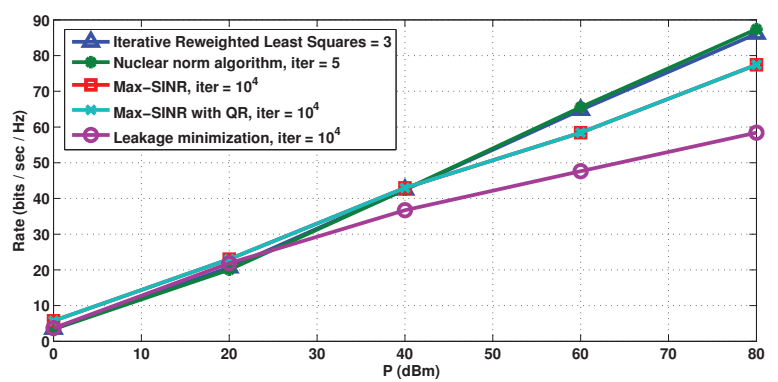

Fig. 4. Sum-rate versus $P$, for a 3 -user MIMO interference channel, $M_{t}=$ $8, M_{r}=4, d=3$

Fig.5, illustrate the variations in the average interference free dimensions with $P$ for $(8 \times 4, d=3)^{3}$ system. It is obvious that our proposed IRLS approach achieves the highest DoF compared with all preceding algorithms at $d=3$. It achieves on average 1.875 DoF with 1.587 achieved by the nuclear norm approach which achieves the nearest performance. The maximum $S I N R$ approach and the leakage minimization approach doesn't achieve more than 1 DoF per user.

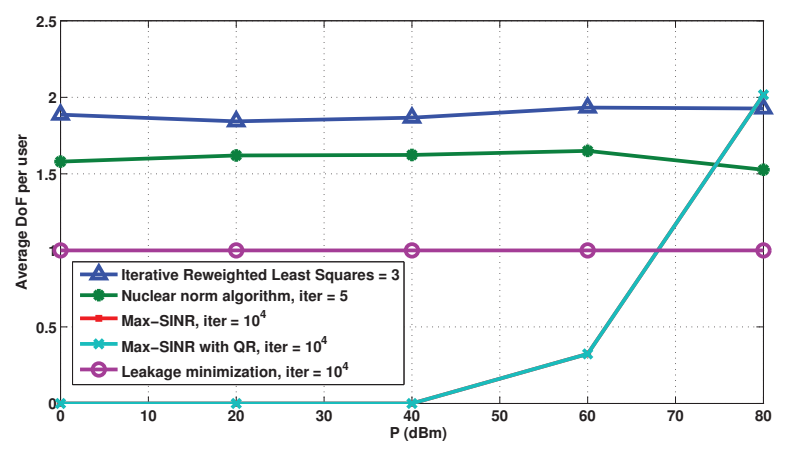

Fig. 5. Average DoF per user versus $P$, for a 3-user MIMO interference channel, $M_{t}=8, M_{r}=4, d=3$

\section{Conclusion}

In this paper, we propose an Iterative Reweighted Least Squares interference alignment algorithm for K-user MIMO 
interference channel. This algorithm follows the rank constrain rank minimization framework to alternatively minimizing both the precoding and postcoding matrices. We have showed that in some cases, the algorithm can meet the conditions for perfect IA. However, when the perfect IA conditions is difficult to achieved, we aim to minimize the rank of subspace spanned by the interference while keeping the signal subspace fullranked. The proposed heuristic was developed based on the IRLS relaxation of the rank cost function introduced in [15]. Furthermore, we have been able to modify the heuristic to cope with the requirements of the IA process. Our results show the effectiveness of the proposed IRLS algorithm in terms of the average sum rate and the average DoF per user.

\section{ACKNOWLEDGEMENT}

The authors would like to thank The ministry of higher education(MoHE)-mission department, and Egypt-Japan University of Science and Technology(E-JUST) for funding our work.

\section{REFERENCES}

[1] V. R. Cadambe and S. Jafar, "Interference Alignment and Degrees of Freedom for the $k$ - user Interference Channel," IEEE Transactions on Information Theory, vol. 54, no. 8, pp. 3425-3441, Aug. 2008.

[2] M. Maddah-Ali, A, Motahari, and A. Khandani, "Communication over MIMO X Channels: Interference Alignment, Decomposition, and Performance Analysis," IEEE Transaction on Information Theory, vol.54, no. 8, pp.3457-3470, Aug. 2008.

[3] Omar El ayach, Steven W. Peters, Robert W. Heath Jr. "The Practical Challenges of Interference Alignment," IEEE Wireless Communications, vol. 20, no. 1, pp. 35-42, Feb. 2013.

[4] Basak Guler and Aylin Yener, "Interference Alignment for Cooperative MIMO Femtocell Networks," Proceedings of IEEE Global Telecommunications Conference, Globecom'11, Houston, Texas, Dec. 2011

[5] Razavi S.M., Ratnarajah T, "Interference alignment in coordinated multipoint systems," Proceeding of of $46_{t h}$ Signals, Systems and Computers, ASILOMAR, pp. 1576 - 1580, Pacific Grove, CA, Nov. 2012.

[6] B. Abdelhamid, M. Elsabrouty, M. Alghoniemy, S. Elramly, O. Muta, and H. Furukawa "Joint Optimization Design for Multiple Underlay MIMO Cognitive Transceiver with Channel Uncertainty," Technical Report of IEICE CS, Vol. 112, No. 484, pp. 235-240, March 2013

[7] B. Abdelhamid, M. Elsabrouty, M. Alghoniemy, S. Elramly, O. Muta, and H. Furukawa, "Joint optimization design for single underlay cognitive transceiver with channel uncertainty," IEICE ComEx, vol.2, no.3, pp.111117, March 2013

[8] S. Akoum, M. Kountouris, M. Debbah and R. W. Heath, Jr., "Spatial Interference Mitigation for Multiple Input Multiple Output Ad hoc Networks: MISO Gains", in Proc. of the IEEE Asilomar Conference on Signals, Systems and Computers (Asilomar), Pacific Grove, CA, Nov. 2011.

[9] C. Yetis, T. Gou, S. Jafar, and A. Kayran, "On the Feasibility of Interference Alignment in MIMO Interference Networks ," IEEE Transactions on Signal Processing., vol. 58, no. 9, pp. 4771-4782, Sep. 2010.

[10] K. Gomadam, V. Cadambe, and S. Jafar, "Approaching the Capacity of Wireless Networks through Distributed Interference Alignment," in Proc. IEEE Global Telecommunication Conf.(GLOBECOM), New Orleans, LA, pp.1-6, Dec. 2008.

[11] S. W. Peters and R. W. Heath Jr., "Interference Alignment via alternative minimization," in Proc. IEEE Int. Conf. Acoust., Speech Signal Processing. (ICASSP), Taipei, Taiwan, pp.2445-2448, Apr. 2009.

[12] K. Gomadam, V. Cadambe, and S. Jafar, "A distributed numerical approach to interference Alignment and applications to Wireless Interference Networks," IEEE Trans. Inf. Theory, vol. 57, no. 6, pp. 3309-3322, Jun. 2011.
[13] H. Yu, Park, Y. Sung, and Y. H. Lee, "A least squares approach to joint beam design for Interference Alignment in Multi-user Interference Channels," IEEE Trans. Signal Processing, vol. 58, no. 9, pp. 4960-4966, Sep.2010.

[14] Dimitris S. Papailiopoulos, and Alexandros G. Dimakis, "Interference Alignment as a Rank Constrained Rank Minimization," IEEE Trans. Signal Processing, vol. 60, no. 8, pp.4278-4288, Aug. 2012.

[15] Karthik Mohan, Maryam Fazel, "Iterative Reweighted Algorithms for Matrix Rank Minimization," Journal of Machine Learning Research (JMLR), vol. 13, pp. 34413473, Nov. 2012.

[16] M. Razaviyayn, M. Sanjabi, and Z. Q. Luo, "Linear Transceiver Design for Interference Alignment: Complexity and Computation," IEEE Trans. Inf. Theory, vol. 58, no. 5, pp. 2896-2910, May 2012.

[17] B. Recht, M. Fazel, and P. Parrilo, "Guaranteed Minimum Rank Solutions of Matrix Equations via Nuclear Norm Minimization," SIAM Rev., vol.52, pp. 471-501, Aug. 2010.

[18] S. W. Peters and R. W. Heath, "Cooperative algorithms for MIMO interference channels," IEEE Transactions on Vehicular Technology, vol. 60, no. 1, pp. 206-218, Jan 2011.

[19] M. Grant and S.Boyd, CVX: MATLAB Software for Disciplined Convex Programming(Web Page and Software)June 2009 [Online]. Available: http://stanford.edu/ boyd/cvx 